\title{
Chest radiography of contemporary trans-catheter cardiovascular devices: a pictorial essay
}

\author{
Subha Ghosh ${ }^{1}$, Mostafa Abozeed ${ }^{2,3}$, Mnahi Bin Saeedan ${ }^{1}$, Subha V. Raman ${ }^{4}$ \\ ${ }^{1}$ Thoracic Imaging, Imaging Institute, Cleveland Clinic, Cleveland, OH, USA; ${ }^{2}$ Cardiopulmonary Imaging Division, University of Alabama at \\ Birmingham, Birmingham, AL, USA; ${ }^{3}$ Radiology Department, Al-Azhar University, Cairo, Egypt; ${ }^{4}$ Indiana University Health and IU Krannert \\ Institute of Cardiology, Indianapolis, IN, USA \\ Contributions: (I) Conception and design: All authors; (II) Administrative support: None; (III) Provision of study materials or patients: None; (IV) \\ Collection and assembly of data: None; (V) Data analysis and interpretation: None; (VI) Manuscript writing: All authors; (VII) Final approval of \\ manuscript: All authors. \\ Correspondence to: Subha Ghosh, MD, MBA. Imaging Institute, Cleveland Clinic Foundation, 9500 Euclid Ave, Cleveland, OH 44195, USA. \\ Email: ghoshs2@ccf.org.
}

\begin{abstract}
There is a plethora of cardiovascular devices used for therapy and monitoring, and newer devices are being introduced constantly. As a result of advancement of medical technology and rapid development of such technology to address unmet needs across cardiovascular care, multiple conditions which were previously treated surgically or with medications now benefit from trans-catheter device-based evaluation and management. Moreover, innovation to existing technology has transformed the structural design of many traditional cardiovascular devices, making them safer and enabling easier deployment within the chest (catheter-based versus surgical). A post-procedure chest radiography (CXR) is often the first routine imaging test ordered in these patients. A CXR is a relatively inexpensive and noninvasive imaging tool, which can be obtained at the patient's bedside if needed. Commonly implanted cardiovascular devices can be quite easily checked for appropriate positioning on routine CXRs. Potential complications associated with malpositioning of such devices may be life-threatening. Such complications often manifest early on CXRs and may not be readily apparent on clinical examination. Prompt recognition of such abnormal radiographic appearances is critical for timely diagnosis and effective management. Clinicians need to be familiar with new devices in order to assess proper placement and identify complications related to mal-positioning. This pictorial essay aims to describe the radiologic appearances of contemporary cardiovascular devices, review indications for their usage and potential complications, and discuss magnetic resonance imaging (MRI) compatibility.
\end{abstract}

Keywords: Cardiovascular devices; trans-catheter; chest radiography (CXR)

Submitted Jul 08, 2020. Accepted for publication Oct 22, 2020.

doi: $10.21037 / \mathrm{cdt}-20-617$

View this article at: http://dx.doi.org/10.21037/cdt-20-617

\section{Introduction}

There is a plethora of newer transcatheter intra-cardiac and vascular devices. Chest radiography (CXR) plays an important role in assessment and follow-up of such devices. It is often the initial imaging test ordered after placement, though many devices are incidentally discovered on CXR.
This pictorial essay aims to highlight the radiographic appearances of relevant, recent cardiovascular devices that are deployed using a percutaneous, trans-catheter technique. We discuss appropriate indications for usage, complications related to abnormal placement, MR compatibility, and closest mimics of each device. 


\section{Classification}

Transcatheter cardiovascular devices can be categorized based on functionality:

(I) Valvular pathologies: transcatheter aortic and pulmonic valve replacement (TAVR, TPVR) and percutaneous management of mitral as well as tricuspid regurgitation (TR) (clip placement);

(II) Congenital heart disease: atrial and ventricular septal occlusion, patent ductus arteriosus (PDA) closure, and endovascular treatment for coarctation of aorta;

(III) Heart failure and post myocardial infarction (MI): ventricular partitioning device is highlighted. Transcatheter ventricular assist devices, intraaortic balloon pumps (IABP) placed via femoral or axillary arteries, and different configurations of extra-corporeal membrane oxygenation cannulae (ECMO) are also shown;

(IV) Monitoring and pacing: leadless cardiac pacemaker, implantable loop recorder, and ambulatory heart monitoring devices are highlighted. An exhaustive list of various routine pacemaker and cardiac defibrillator devices as well as the now common Swan-Ganz catheter are not included in this review, though some newer lead configurations such as Bundle of His leads which are positioned towards the IVS are shown;

(V) Prevention of thromboembolism in the systemic and pulmonary circulation: Percutaneous left-atrial appendage closure and cava filters.

\section{Discussion}

An increasing array of cardiovascular diseases are amenable to trans-catheter management, and newer trans-catheter devices are being constantly introduced or modified. Continuously improving efficacy and safety of these procedures have widened the target population to include not only candidates who are medically unfit for surgery but also selected patients who have no contraindications for surgery. Trans-catheter procedures are less invasive, with many offering comparable efficacy when compared to surgery (1). Some procedures such as trans-catheter valve replacement and closure of septal defects have become commonplace in interventional cardiology, while others such as left ventricular partitioning device placement, are relatively uncommon.

CXR is often the initial imaging screening test to identify and evaluate such devices, and thus plays a crucial role. It can provide easy confirmation of optimal device placement and help in early detection of potentially grave complications related to abnormal placement before these are manifested clinically. Imagers should therefore be familiar with these devices, their proper imaging appearances and potential complications. Malpositioning, migration/embolization and fracture/deformity of cardiovascular devices, as well as postprocedure complications such as pneumothorax (Figure 1A), pneumomediastinum (Figure $1 B$ ), pneumopericardium (Figure 1C), and hemorrhage within pleural (Figure 1D), mediastinal (Figure 1E), or pericardial compartments (Figure $1 F$ ) resulting in new/enlarging/loculated pleural effusions, cardio-mediastinal widening, and pericardial effusion are radiographically-detectable. There are inherent limitations of the CXR in detecting all potential complications related to such devices. Several devicespecific complications such as infection, thromboembolism, cardiac arrhythmias, myocardial or vessel wall trauma, etc., are not detectable by CXR. We emphasize that clinical suspicion of such radiographically-occult complications should prompt further imaging with echocardiography or computed tomography (CT).

Echocardiography and CT are not indicated as routine screening tests for device placement though may serve as important problem solving tools to confirm abnormal placement and further evaluate suspected complications. For example, CXR obtained after a pacemaker implantation can suggests abnormal position of a pacemaker lead outside the cardiac borders. A subsequent echocardiogram can show tamponade from myocardial perforation, while CT is able accurately pin point the abnormal location of the lead, site of tear, etc. A magnetic resonance imaging (MRI) scan may be indicated in patients with indwelling devices for evaluating underlying cardiac or other unrelated diseases. This may prompt a screening CXR to "clear" these patients for MRI, and thus knowledge of MR compatibility of each device is important.

Trans-catheter intra-cardiac and vascular devices have characteristic radiographic appearances. Several of these devices remain connected to long intra-arterial or intravenous catheters, with a portion projecting into the cardiac chambers. Examples include temporary transcatheter ventricular assist device or Impella (tip inside the ventricular cavity), IABP (tip within the proximal descending aorta), 

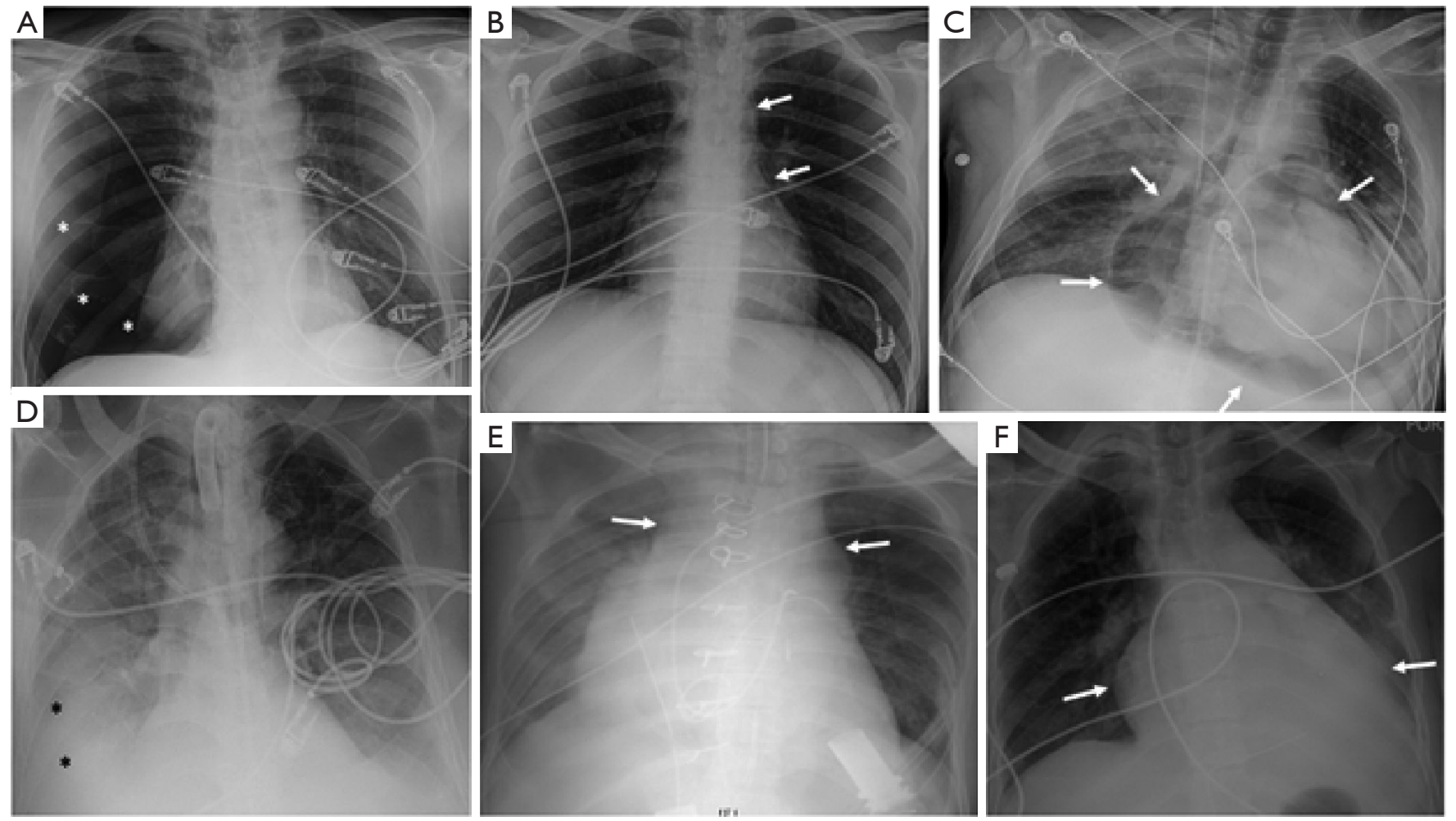

Figure 1 Radiographically-detectable post-procedure complications. (A) Right pneumothorax (asterisks). (B) Pneumomediastinum (arrows). (C) Pneumopericardium (arrows). (D) Pleural effusions due to intrapleural hemorrhage (asterisks). (E) Mediastinal widening due to mediastinal hematoma (arrows). (F) Pericardial effusion (enlarged cardiac silhouette, arrows).

ECMO cannulae (tip in the vena-cavae or right atrium for veno-venous, and in a central or peripheral artery and vein in veno-arterial), and Swan-Ganz catheters (tip in a central pulmonary artery- usually the right). Cardiac pacemakers and defibrillators with chest-wall pulse-generator and intracardiac leads are fairly common, though a few newer lead positions (Bundle of His) may not be routinely encountered.

Most other relevant transcatheter devices have one of few distinct shapes, namely—stent-like, short clip-like, capsular appearance and clamshell, umbrella or parachute shaped. The smallest of the reviewed devices (CardioMEMS) has a unique figure-of-eight shape.

Some of these devices are seen in characteristic locations such as the SVC area, distal aortic arch and aortopulmonary window region, which make them easier to recognize on a single view radiograph (frontal or PA). Other devices which project over specific chambers, septae or valves, can be difficult to accurately localize on a single frontal or PA view without the help of a lateral CXR.

\section{Valvular pathologies}

\section{Transcatheter aortic valve devices}

\section{Clinical indications}

Since 2002, TAVR has been validated as a suitable alternative to surgical aortic valve replacement in selected patients with severe aortic stenosis considered non-operable or intermediate- high risk for surgical repair $(2,3)$.

\section{Device make and radiographic appearance}

Commercially available TAVR systems include the Sapien 3 (Edwards Lifesciences, Irvine, CA), Sapien XT (Edwards Lifesciences, Irvine, CA), CoreValve (Medtronic Inc., Minneapolis, MN), and Portico (St Jude Medical or Abbott Inc., Chicago, IL) trans-catheter aortic heart valves. The Sapien 3 valve (Figure $2 A, B, C$ ) and Sapien XT valve have similar constructions and use bovine pericardial tissue fixed to a cobalt chromium alloy frame and capped with a polyethylene outer skirt. The CoreValve is made from porcine cardiac tissue and self-expanding nitinol frame 
A
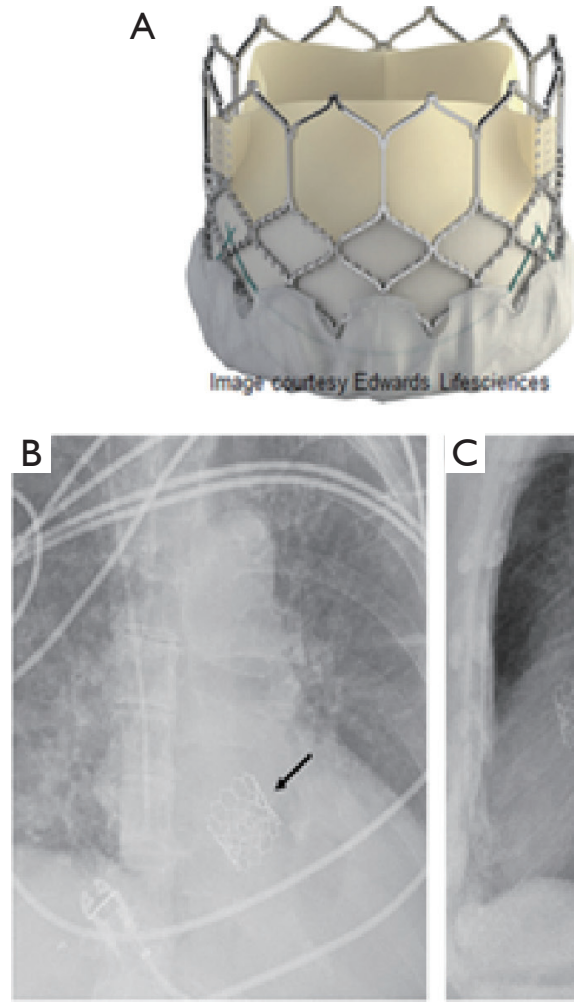

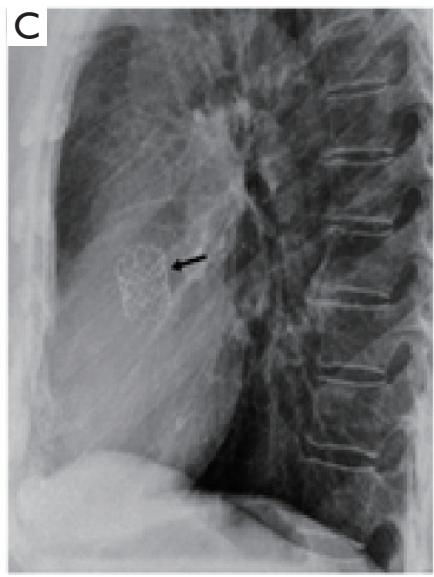

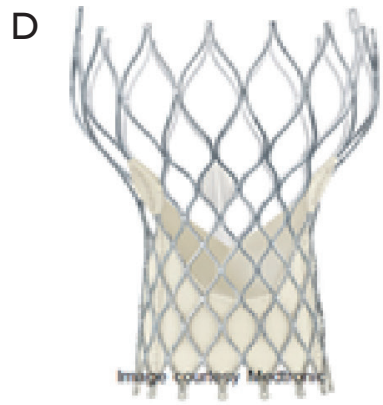

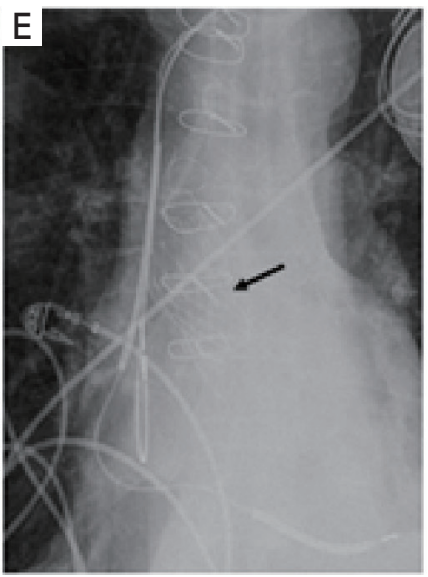

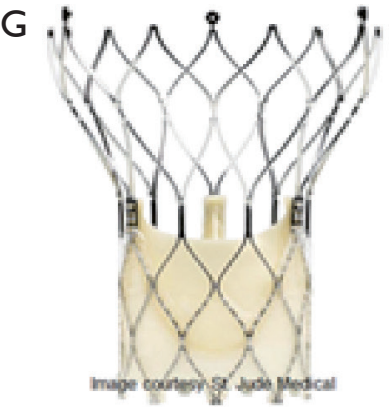

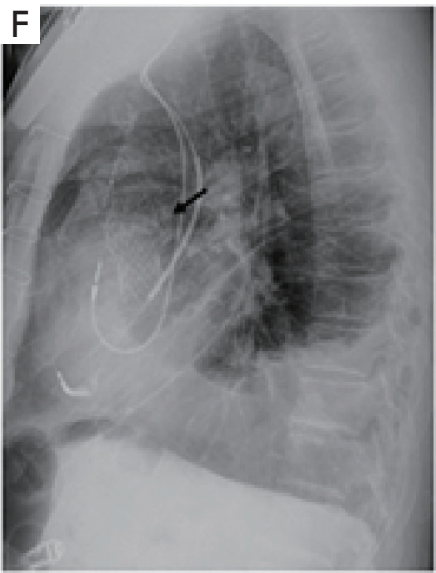

Figure 2 Sapien 3, CoreValve, and Portico transcatheter aortic valves. (A) Photograph shows the Sapien 3 valve. (B) Frontal and (C) lateral radiographs show the Sapien 3 valve (arrow) in the expected aortic position. (D) Photograph shows the CoreValve. (E) Frontal and lateral (F) radiographs show the implanted CoreValve (arrow) in the expected aortic valve position. (G) Photograph shows the Portico valve.

(Figure 2D,E,F). The Portico valve has bovine leaflets with a porcine sealing cuff and flared self-expanding stent (Figure 2G). On CXRs, each of these trans-catheter valves with their different designs are localized to the aortic valve position (4).

\section{Device-specific complications}

Annular rupture (i.e., injury to the aortic root and/or left ventricular outflow tract) is the most severe complication. Stent fracture and device malposition, migration and/or embolization are other potential complications $(5,6)$.

\section{Transcatheter pulmonary valve devices}

\section{Clinical Indications}

TPVR is used for pulmonic regurgitation or pulmonic stenosis $(7,8)$.

\section{Device make and radiographic appearance}

TPVR devices are usually made from bovine or porcine tissue and multiple diameters (sizes) are available. Melody pulmonic valve (Medtronic Inc., Minneapolis, MN) is a commercially available trans-catheter pulmonary valve and consists of a bovine jugular venous conduit that is attached to a balloon-expandable platinum stent (Figure 3A) (9). On CXRs, the Melody valve metallic stent should be projected in the position of the pulmonic valve (Figure 3B).

\section{Device-specific complications}

Non visualization of a Melody valve in the expected position may indicate malposition, migration, or embolization. Stent fracture can also be noted at radiography. Additional complications include paravalvular leak, pulmonary artery pseudo-aneurysm and pulmonary hemorrhage (10).

\section{Percutaneous mitral regurgitation therapy (mitral clips)}

\section{Clinical indications}

Percutaneous mitral valve repair using the MitraClip system (Abbott Structural Heart, Santa Clara, CA) creates a double 

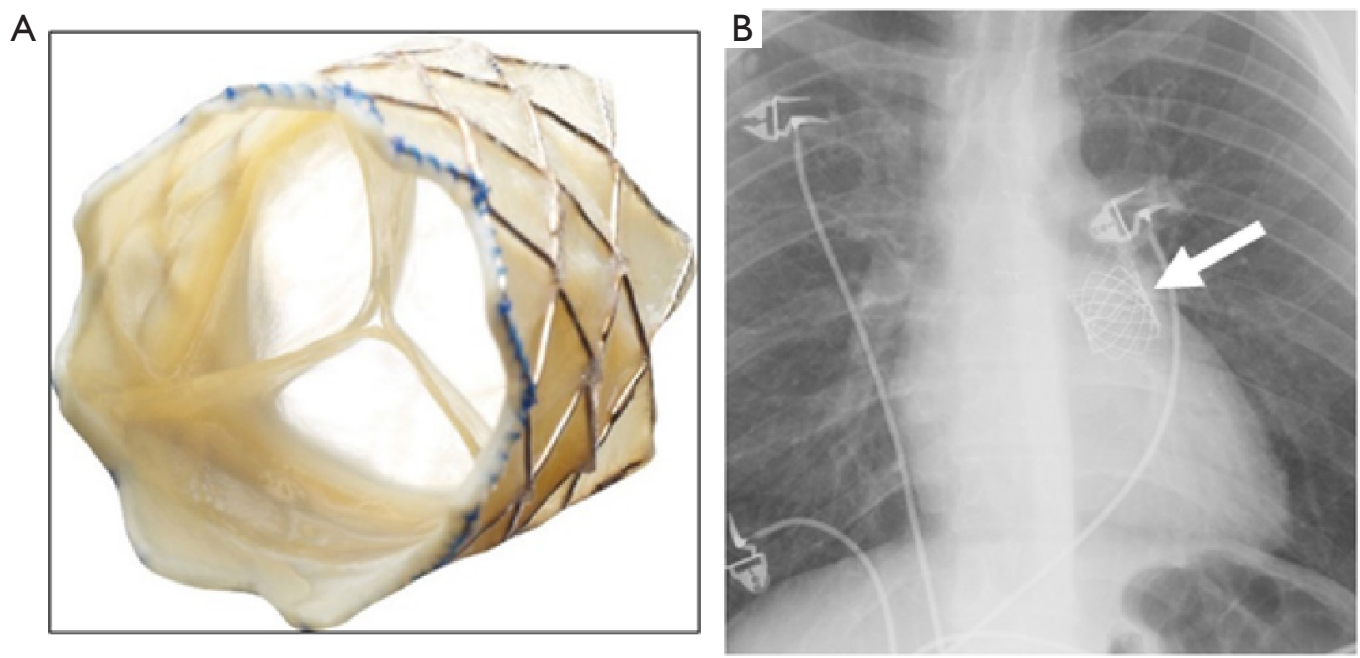

Figure 3 Melody TPVR device. (A) Photograph shows the Melody TPVR device (Image courtesy Medtronic). (B) Frontal chest radiograph shows a melody TPVR device in the expected pulmonic position.
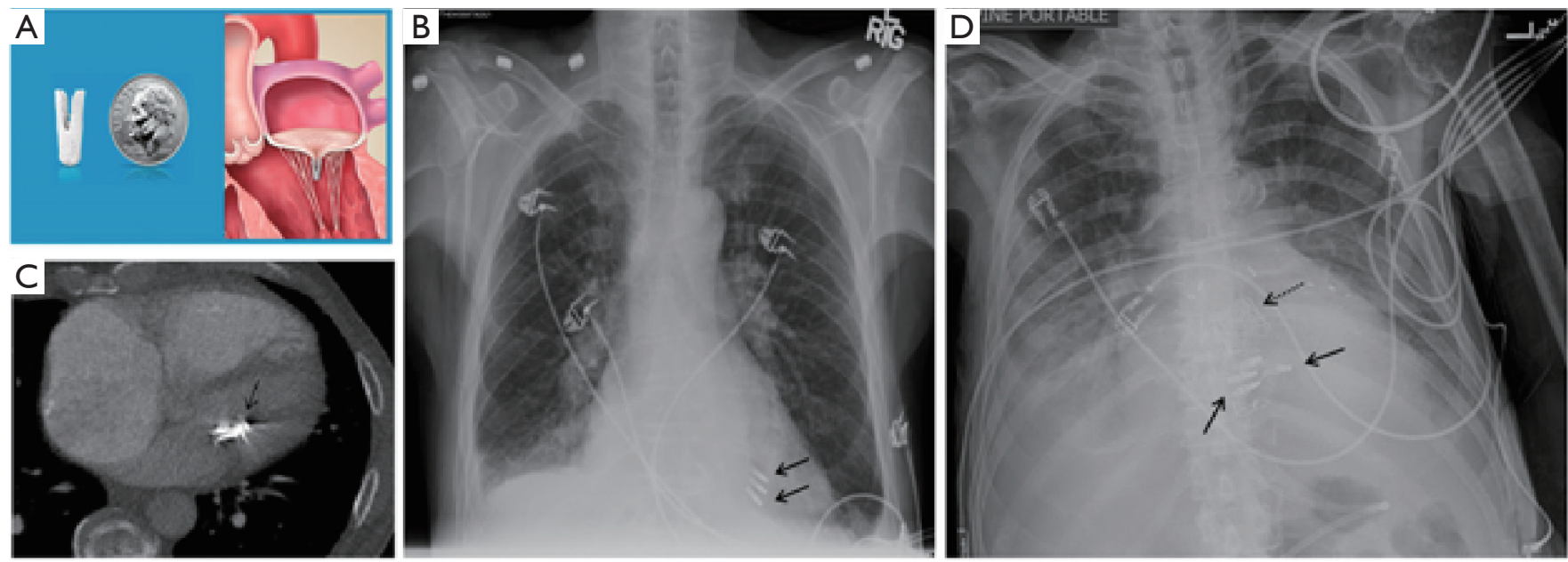

Figure 4 MitraClip. (A) Photograph of the MitraClip device shows the device size and the adjacent illustrated diagram of the LVOT shows the mitral valve and seated device (Image courtesy Abbott). (B) Frontal chest radiograph shows MitraClips (arrows) that are projected side by side parallel to one another over the expected position of the mitral valve and appropriately positioned. (C) Axial CT image of the same patient shows the clips causing metallic artefacts (arrow) over the mitral valve leaflets. (D) Frontal chest radiograph shows MitraClips (arrows) that have been used for tricuspid regurgitation and project over the midline tricuspid location. TAVR is also noted (dashed arrow).

orifice and has been successfully used in selected patients with functional or degenerative mitral regurgitation (11).

\section{Device make and radiographic appearance}

The MitraClip is a polyester-covered cobalt chromium clip. Two adjacent metallic clips in the expected mitral position are seen on radiography (Figure $4 A, B, C)$ (4).

\section{Device-specific complications}

Device complications include injury to the mitral valve, partial clip detachment and embolization, device migration (non-mitral position), and mitral stenosis $(12,13)$. 


\section{Percutaneous TR therapy (tricuspid clips)}

\section{Clinical indications}

Symptomatic TR has been treated with off-label use of MitraClip system, though clips specifically designed for the tricuspid valve (TriClip) have recently been FDA approved to treat symptomatic severe TR (Abbott Structural Heart, Santa Clara, CA) (14).

\section{Device make and radiographic appearance}

Similar to the MitraClip though in a tricuspid location (midline on frontal CXR, Figure 4D).

\section{Mimic}

Per-oral endoscopic myotomy clips used for endoscopic management of achalasia may look similar to MitraClip or Triclip. The myotomy clips project either midline or to the right of midline in keeping with the anatomic location of a dilated thoracic esophagus.

\section{Congenital heart disease}

\section{Atrial septal defect (ASD) occlusion device}

\section{Clinical indication}

These are indicated in ASD with right atrial and right ventricular enlargement, left-to-right shunting, prevention of paradoxical embolisms, and documented platypneaorthodeoxia syndrome. Only the medium to small secundum ASDs are amenable to percutaneous closure $(15,16)$.

\section{Device make and radiographic appearance}

The Amplatzer Septal Occluder (St Jude Medical or Abbott Inc., Chicago, IL) is a catheter-based ASD closure device. It is composed of self-expanding circular double-disk nitinol mesh that snugly occupies the defect with the disc rims closing both sides of the septal wall (Figure 5A) (17). Multiple sizes are available and it may also be used for ventricular septal defect (VSD) closure. On radiographs, the rounded nitinol disks should be projected over the expected site of the interatrial septum or over the interventricular septum in cases of VSD closure (Figure 5B,C,D).

\section{Device-specific complications}

Device embolization is suggested if the device is not seen over its expected location either in the interatrial or interventricular septal region. Residual shunts seen on echocardiography and thromboembolic events are potential complications of a failed procedure. Device infections, arrhythmias and cardiac perforations (rare) can also occur.

\section{Patent ductus arteriosus (PDA) closure devices}

\section{Clinical indications}

Trans-catheter occlusion of PDA may be indicated for nonsurgical patients with left ventricular volume overload, patients with pulmonary artery hypertension $(\mathrm{PAH})$ but with pulmonary artery pressure (PAP) $<2 / 3$ of systemic pressure or pulmonary vascular resistance (PVR) less than $2 / 3$ of systemic vascular resistance (SVR), and small PDAs with continuous murmur (18).

\section{Device make and radiographic appearance}

Multiple devices such as Amplatzer duct occluder II (ADO) devices (St Jude Medical or Abbott Inc., Chicago, IL) (Figure 6), Cera occluder device, and coils have been used. For larger defects other devices include Cardi-O-Fix device, Cocoon device and muscular septal defect occluder. Recently, a newer device (Occlutech occluder, Occlutech Germany) was introduced with designs to overcome some of the limitations of the ADO device. These devices are seen in the aorto-pulmonary window on radiography $(19,20)$.

\section{Device-specific complications}

Device embolization, infection, narrowing of the aortic isthmus and left pulmonary artery, hemolysis, spontaneous recanalization, and post-procedure left ventricular systolic dysfunction are potential complications (21).

\section{Endovascular treatment for coarctation of aorta}

\section{Clinical indication}

A primary therapeutic option for many adults and adolescents with coarctation of the aorta, or re-coarctation at the site of prior repair, is intravascular stent therapy (22).

\section{Device make and radiographic appearance}

The most often used devices are balloon-mounted stents such as Palmaz (Cordis, Miami Lakes, FL) or Cheatham Platinum (NuMed, Hopkinton, NY) $(18,19,23,24)$. On CXRs, the slightly curved metallic stent is visualized in the expected location of the coarctation, along the distal aortic arch/proximal descending aorta (Figure 7), and rarely characteristic rib notching may be seen (due to chest wall collateral circulation). 

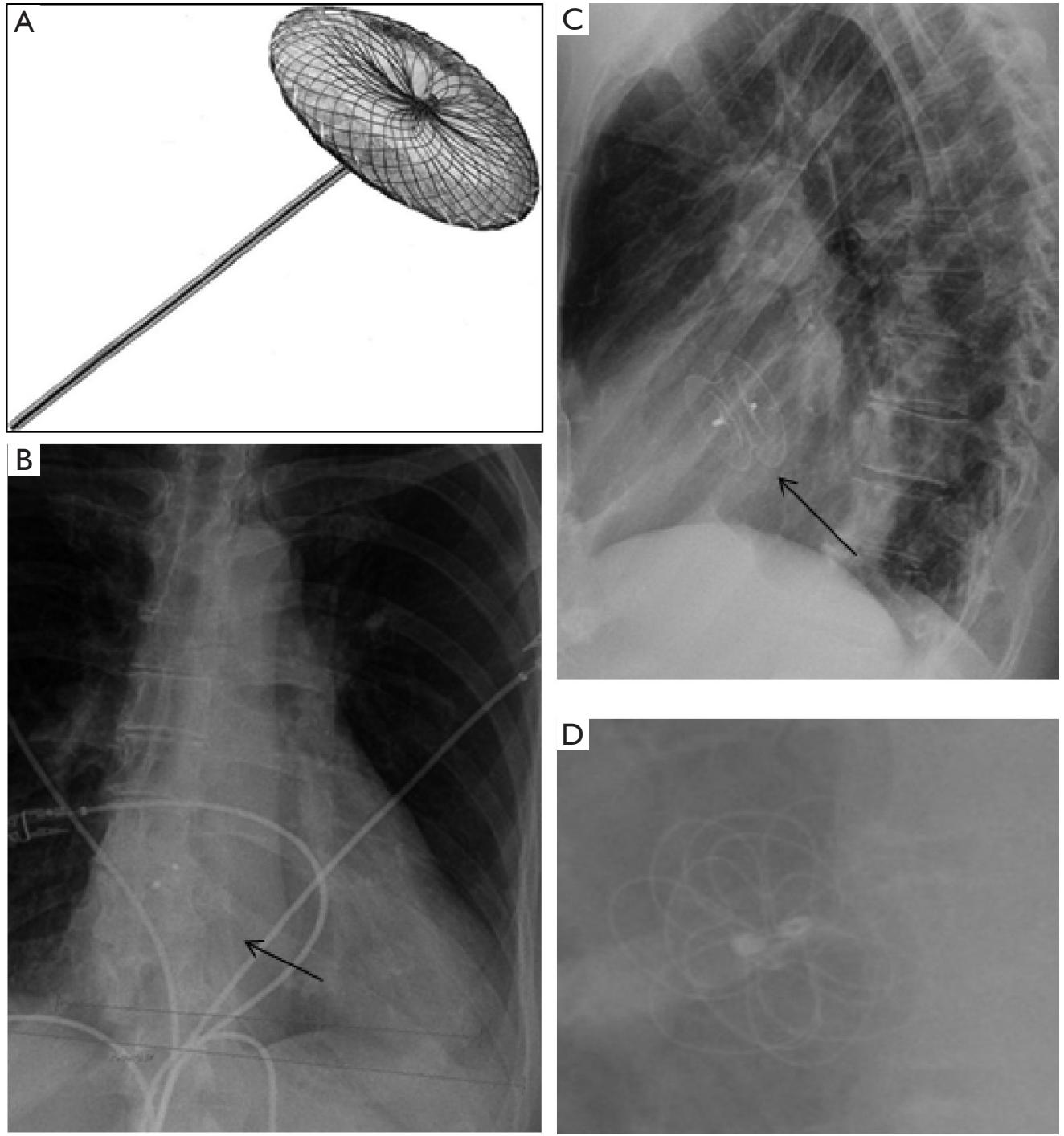

Figure 5 Amplatzer Septal Occluder. (A) Photograph shows the device with self-expanding nitinol mesh (Image courtesy St Jude Medical). (B) Frontal, (C) lateral and (D) magnified chest radiographs show the device (arrow) projected over the expected location of the interatrial septum.

\section{Device-specific complications}

Stent fracture is well recognized but usually does not lead to adverse outcome. Recurrent narrowing is rare. Stent migration during implantation is a potential complication, due to stent slippage or balloon rupture. True late migration may be extremely rare, although fragment distal embolization can be seen with circumferential stent fracture $(25,26)$.

\section{Heart failure and post MI}

Ventricular Partitioning Device (Cardiokinetix, Menlo Park, CA)

\section{Clinical indication}

To preserve normal left ventricular morphology and prevent post injury MI remodeling, reduce left ventricular end diastolic volume, and isolate nonfunctioning from 

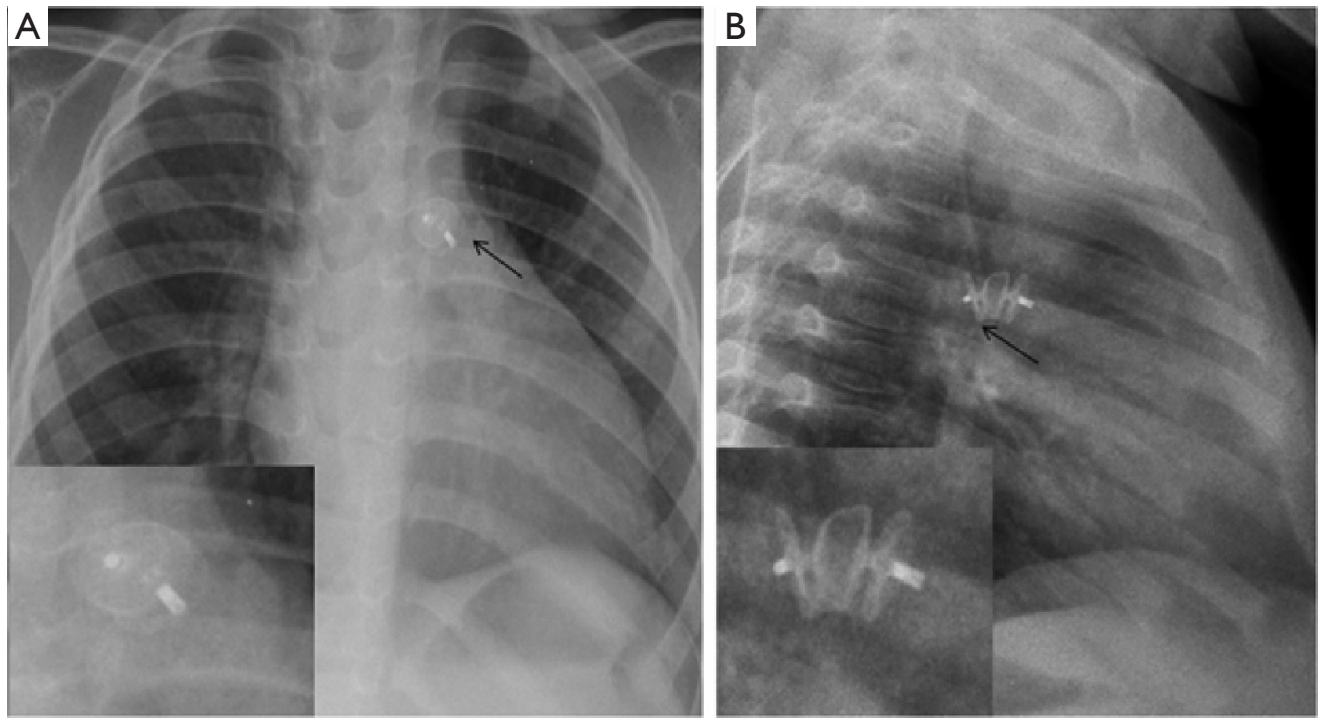

Figure 6 PDA occluder/Amplatzer duct occluder II Additional Sizes (ADO II AS). (A) Frontal and (B) lateral chest radiographs show the device (arrow) over the expected location of the aorto-pulmonary window with magnified images (inserts) that show the discs with tightly woven mesh.
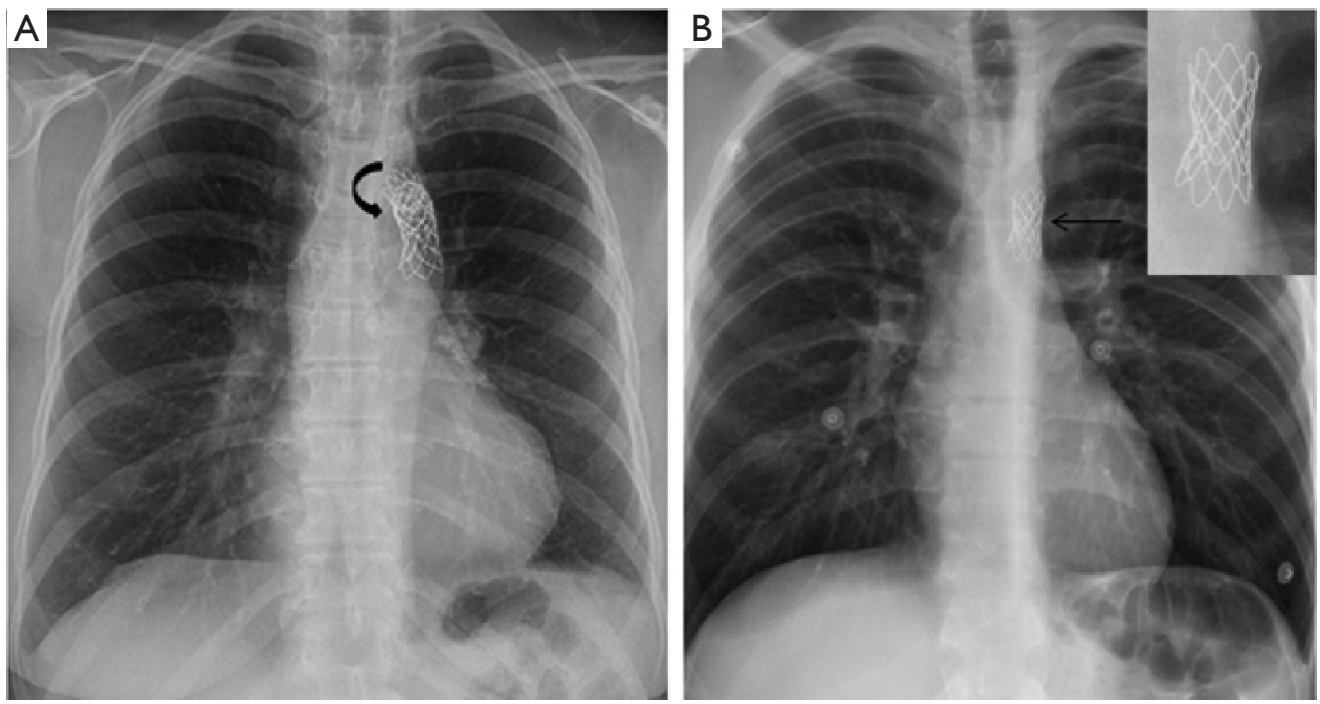

Figure 7 Coarctation of aorta treated with stent. (A) Frontal chest radiograph shows the stent (curved arrow) in the expected position of proximal descending aorta. (B) Frontal chest radiograph with magnified image (insert) in a different patient shows the stent (arrow) projected in the expected location.

functioning myocardium (27).

\section{Device make and radiographic appearance}

The parachute-shaped or umbrella-like device was developed to restore left ventricular function in patients with heart failure and LV apical aneurysm after MI. It is placed percutaneously, and composed of fluoropolymer membrane fitted to an umbrella-shaped nitinol frame. The device "foot" should be seated at the left ventricular apex (Figure 8) on radiographs (28). 

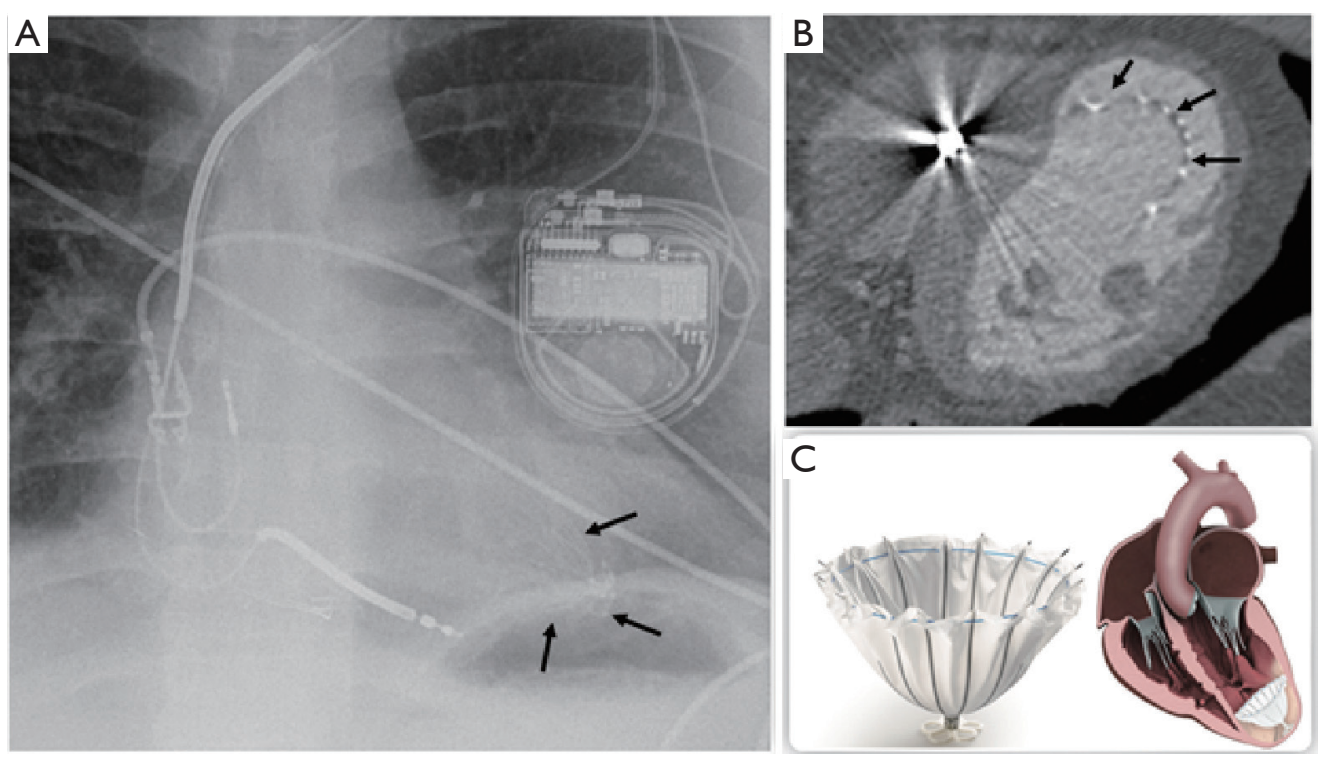

Figure 8 Parachute ventricular partitioning device. (A) Frontal chest radiograph shows the parachute device in the left ventricular apex with wire struts (arrows). (B) Axial CT image at the level of the device shows the wires (arrows) within the aneurysmal left ventricle apex. (C) Photograph and illustration show the device, the nitinol frame, and the ideal positioning of the device at the cardiac apex (image courtesy CardioKinetics).

\section{Device specific complications}

Device malposition or migration are possible complications. Thromboembolism may contribute to a reported stroke rate of $15.9 \%$ at 3 years (29).

\section{Catheter based devices for mechanical circulatory support}

The Impella (Abiomed) is a transcatheter ventricular assist device used for short-term mechanical circulatory support in the setting of cardiogenic shock. It comprises of an inflow (impeller) which draws in blood from the ventricle and an outflow into the ascending aorta. It is inserted percutaneously through the femoral (or axillary) artery and advanced retrograde through the aorta with its bent tip snugly inside the left ventricular cavity (Figure 9). A similar device (Impella RP) can be used for right-sided or biventricular failure (4). The Impella device is unsafe for MRI imaging (30).

IABP counterpulsation is a form of temporary mechanical hemodynamic support. It comprises of a long catheter inserted percutaneously through the femoral (or axillary) artery and advanced retrograde through the aorta with its tip in the proximal descending thoracic aorta. The distal radiopaque marker should project over the proximal descending aorta (Figure 10) (31). This is unsafe for MR imaging (30).

ECMO is used for cardiorespiratory support and can be of two types: veno-venous (VV) and veno-arterial (VA). VV is primarily used for gas exchange (respiratory support) and VA provides both gas exchange and hemodynamic support. CXR shows wide bore venous catheters in the vena-cavae or right atrium for $\mathrm{VV}$ or in a central vein and artery/aorta for VA configurations (Figure 11) (31).

The most serious complications related to these catheter based devices include injury to vessel wall, myocardial wall perforation, thrombus formation and catheter fracture. Disruption of the mitral valve apparatus with resultant severe mitral regurgitation has also been reported with the Impella.

\section{Monitoring and pacing}

\section{Leadless cardiac pacemakers}

\section{Clinical indications}

Variety of cardiac arrhythmias and morphologic abnormalities are considered as indication for cardiac pacing (32). Newly developed leadless pacemakers can be 

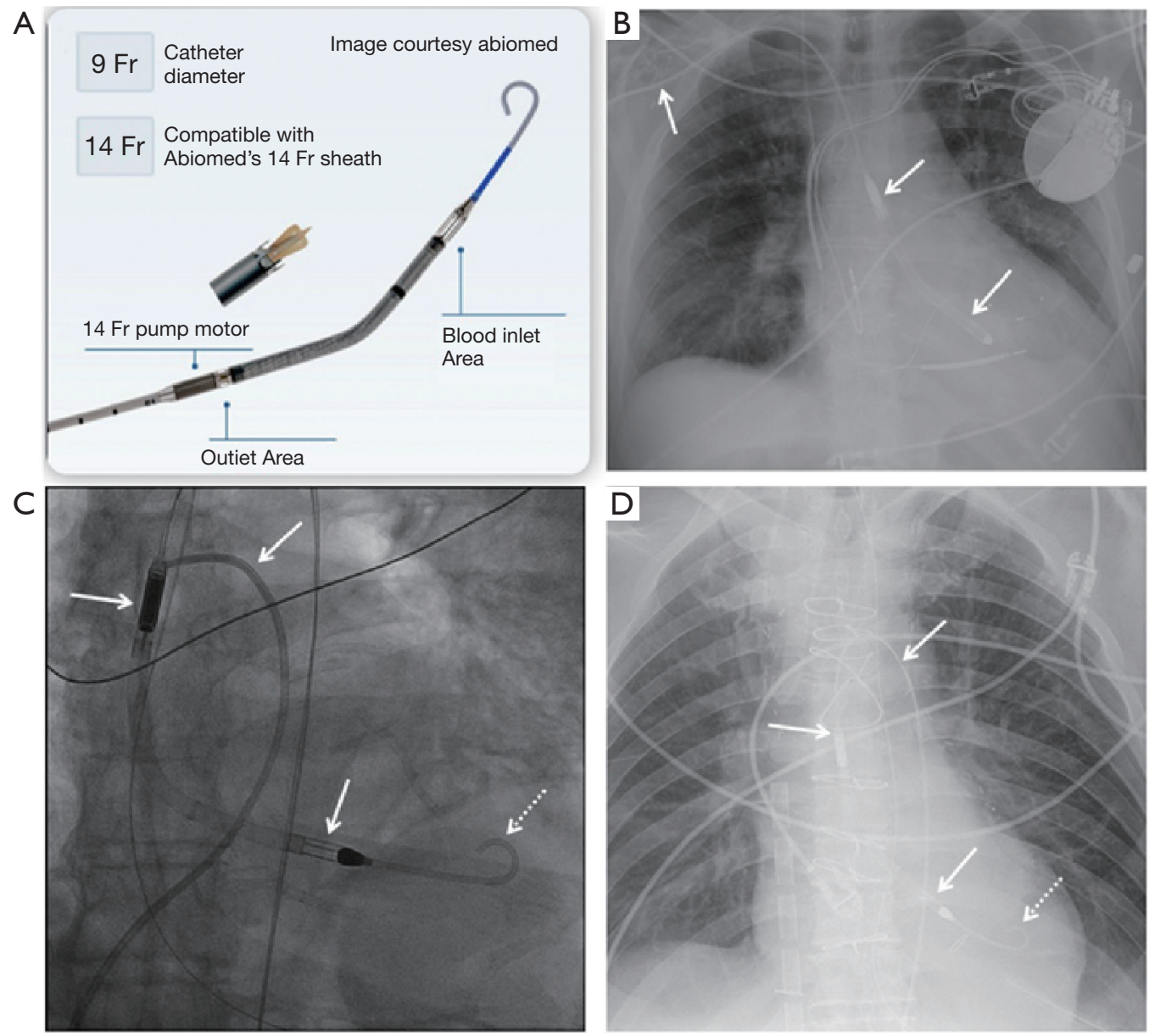

Figure 9 Impella. (A) Impella design with parts. The device draws blood from the LV through the impeller with outflow into the ascending aorta. Similar device (Impella RP) can be used for right-sided or biventricular failure with inflow in the inferior vena cava and outflow tip within the main PA. (B) Frontal chest radiograph shows Impella placed via the right axillary artery with its tip in the left ventricle (LV) (arrows). (C,D) Digital radiograph at the time of insertion (C) and frontal chest radiograph post insertion (D) show placement of Impella (arrows) via the femoral approach. This is inserted percutaneously via the femoral artery, and passed retrograde through the aorta and aortic valve into the left ventricle (LV). Tip of the Impella should project centrally over the LV (dashed arrows).

used for patients who meet the criteria for right ventricular pacing. These devices have the advantage of avoiding leadassociated complications such as lead dislodgement or fracture, cardiac perforation, or venous thrombosis and allow for reliable performance and improved safety $(33,34)$.

\section{Device make and radiographic appearance}

The Nanostim Leadless Pacemaker System (St. Jude Medical or Abbott Inc., Chicago, IL) and the Micra Transcatheter Pacing System (Medtronic Inc., Minneapolis, $\mathrm{MN}$ ) are the current leadless pacemakers. Both are delivered percutaneously to the right ventricle and are transfixed to the myocardium at the apex.

They have screw-in fixation electrode, or multiple tiny nitinol tines that hold the devices in place. The Micra device appears as small battery-shaped electronic device overlying the expected location of the right ventricular apex (Figure 12) on radiography (4).

\section{Device-specific complications}

Device complications include cardiac perforation and thromboembolism $(33,34)$. 

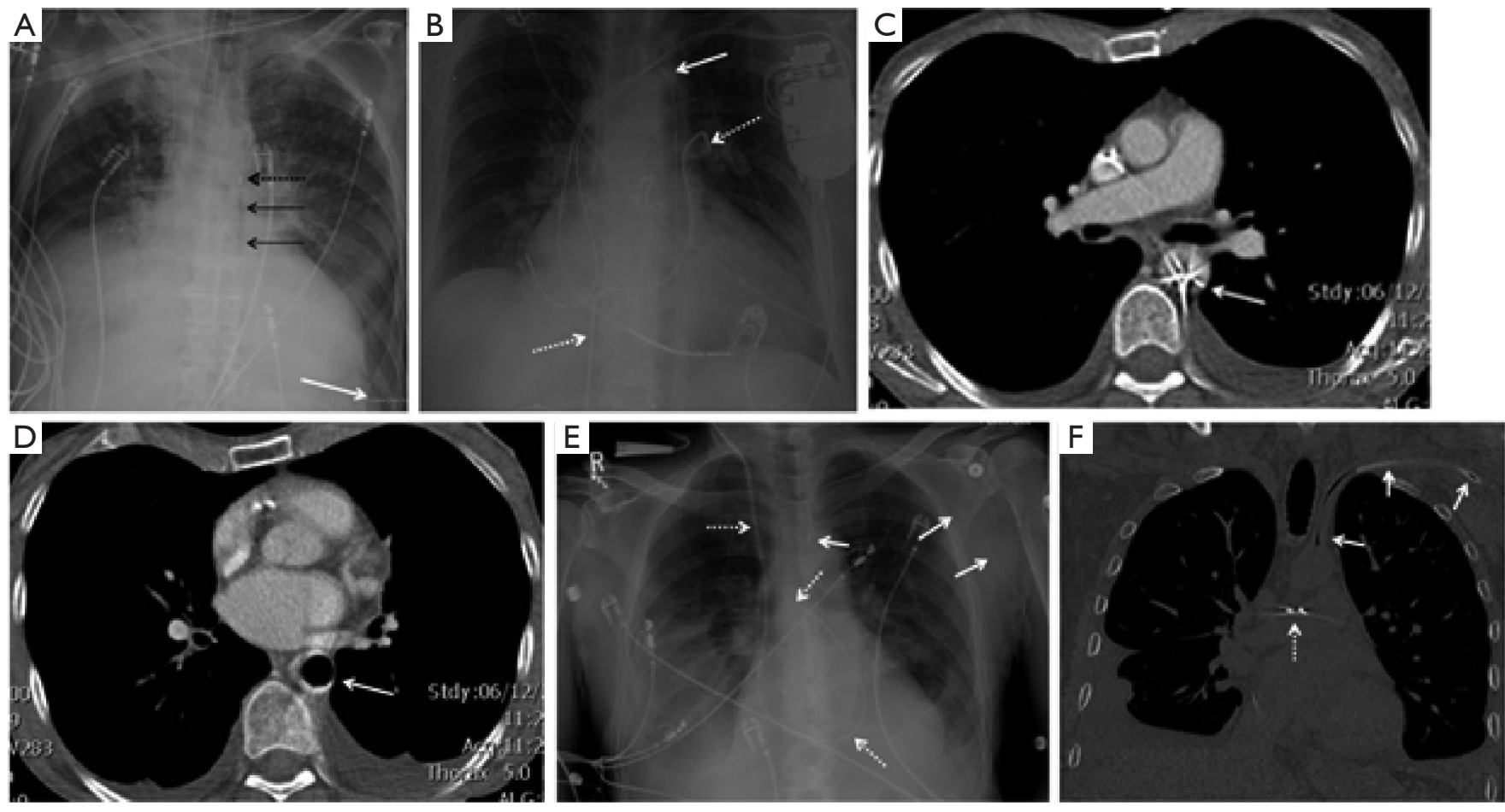

Figure 10 IABP. (A) Frontal chest radiograph shows IABP inserted via the femoral artery with radio-opaque marker tip projected in the proximal descending thoracic aorta in a satisfactory position (dashed arrow). Note, the inflated balloon which appears as a midline long segment lucency below the radiopaque marker (black arrows), is usually not visualized on CXR. It inflates during diastole (promotes coronary filling) and deflates during systole (promotes forward flow). Note asymmetric lucency along the left costophrenic sulcus (white arrow) suggestive of left pneumothorax (“deep sulcus sign”). (B) Frontal chest radiograph shows IABP tip is too far distal and projects above the roof of the aortic arch (arrow), possibly wedged inside the lumen of the proximal left common carotid artery. This should be retracted to prevent intimal damage to the artery and potential stroke. Note, a Swan-Ganz catheter inserted via the femoral vein with its tip in the left pulmonary artery (dashed arrows). (C,D) Contrast-enhanced chest CT shows IABP with tip in the mid-descending thoracic aorta (arrow in C) and balloon inflated just above the diaphragmatic hiatus (arrow in D). This is a suboptimal position and risks mesenteric ischemia due to intermittent occlusion of the celiac and superior mesenteric arteries. (E) Frontal chest radiograph shows IABP inserted via the left axillary artery with marker projected just below the roof of the aortic arch and introducer sheath in the left axillary/subclavian region (arrows). Note, a Swan-Ganz catheter is also present with its tip in the right pulmonary artery (dashed arrows). (F) Chest CT shows the introducer/sheath in the left axillary/subclavian artery and balloon of the IABP (air lucency) in the left subclavian artery (arrows). Note, the radiopaque tip of the Swan-Ganz catheter in the right pulmonary artery (dashed arrows). IABP, intra-aortic balloon pump.

\section{Leadless pacemaker mimic: implantable loop recorder}

\section{Clinical indication}

Implantable loop recorders (Medtronic Inc., Minneapolis, $\mathrm{MN}$ ) are subcutaneously implantable cardiac monitors to continuously record the cardiac rhythm in patients with unexplained palpitations or syncope (35).

\section{Device make and radiographic appearance}

The older device simulates a USB flash-drive, though newer designs are more slender and can be mistaken for a leadless pacemaker on a single frontal-view CXR. The leadless pacemaker has a small dense pointed projection on the tip (Figure 12A,C). The lateral-view CXR clinches the diagnosis as the loop recorder is projected in the subcutaneous chest wall (Figure 13).

\section{Device specific-complications}

Device complications include pain and infection at the site of implantation. Migration is a potential complication. 

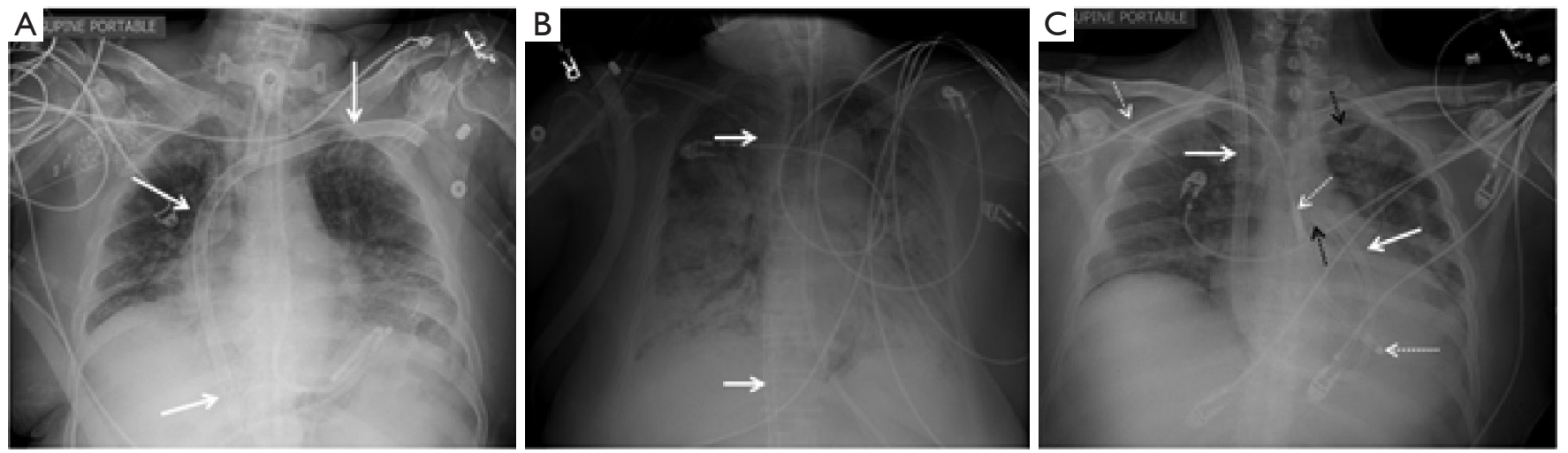

Figure 11 ECMO. (A) Frontal chest radiograph shows VV ECMO with a single wide bore venous cannula which has been inserted via the left subclavian vein, traverses the SVC and right atrium with its tip in the IVC (arrows). (B) Frontal chest radiograph shows VV ECMO with 2 separate cannulas, one inserted via the right internal jugular vein with tip in the upper right atrium and the other inserted via the femoral vein with tip in the mid right atrial cavity (arrows). Note dense bilateral airspace consolidations compatible with diffuse alveolar damage. (C) Frontal chest radiograph shows VA ECMO with venous limb inserted via the right internal jugular vein and arterial limb anastomosed to the aorta (arrows). Note also the presence of an Impella inserted via the right axillary artery with tip in the LV (dashed white arrows), and a Swan-Ganz catheter placed via the left subclavian vein with tip in the right pulmonary artery (dashed black arrows). ECMO, extra-corporeal membrane oxygenation cannulae.
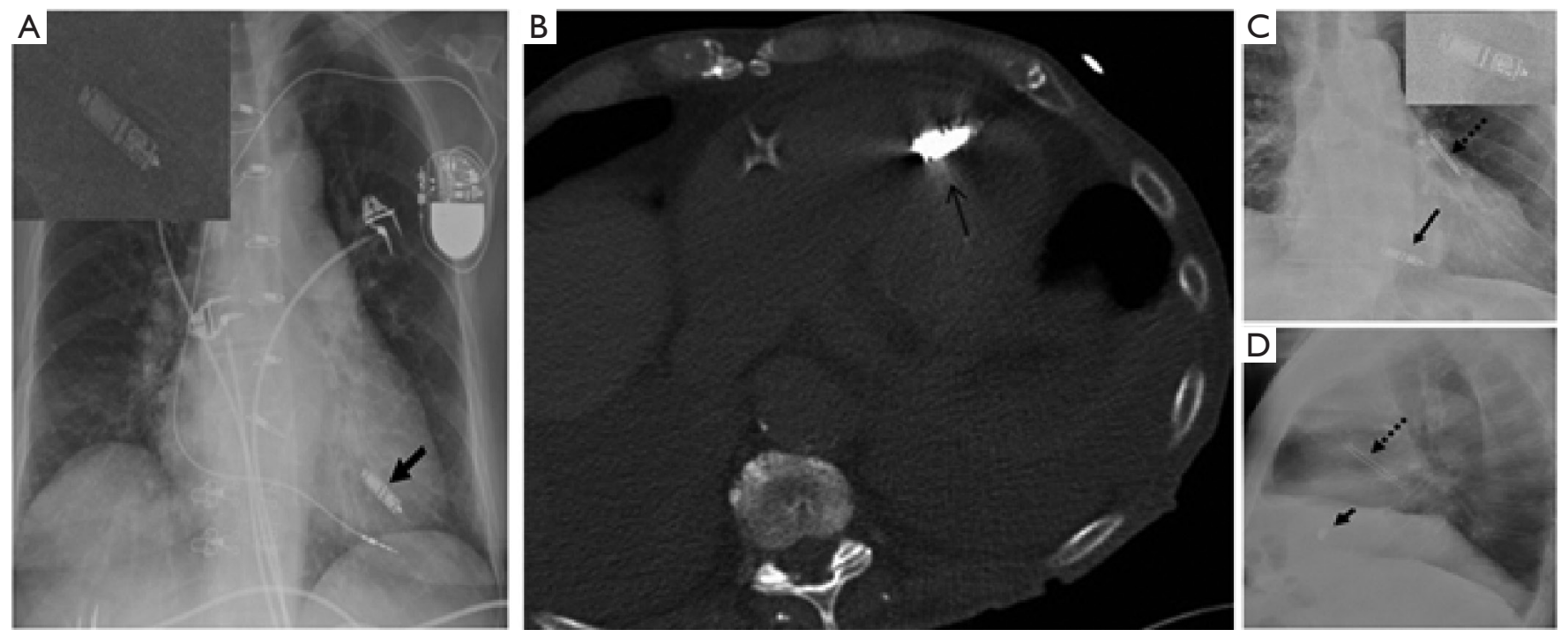

Figure 12 Micra leadless pacemaker. (A) Frontal chest radiograph shows implantable leadless pacemaker (arrow) with magnified image (insert) projecting over the expected location for the device in the right ventricle. (B) Axial CT of a different patient at the level of the interventricular septum shows the well-seated device in the right ventricle. (C) Frontal chest radiograph with magnified image (insert) and (D) lateral chest radiograph show the device in the expected right ventricle position (arrows) and internal fabric details in the magnified image. LAA ligation clip is noted (dashed arrows). LAA, left atrial appendage. 

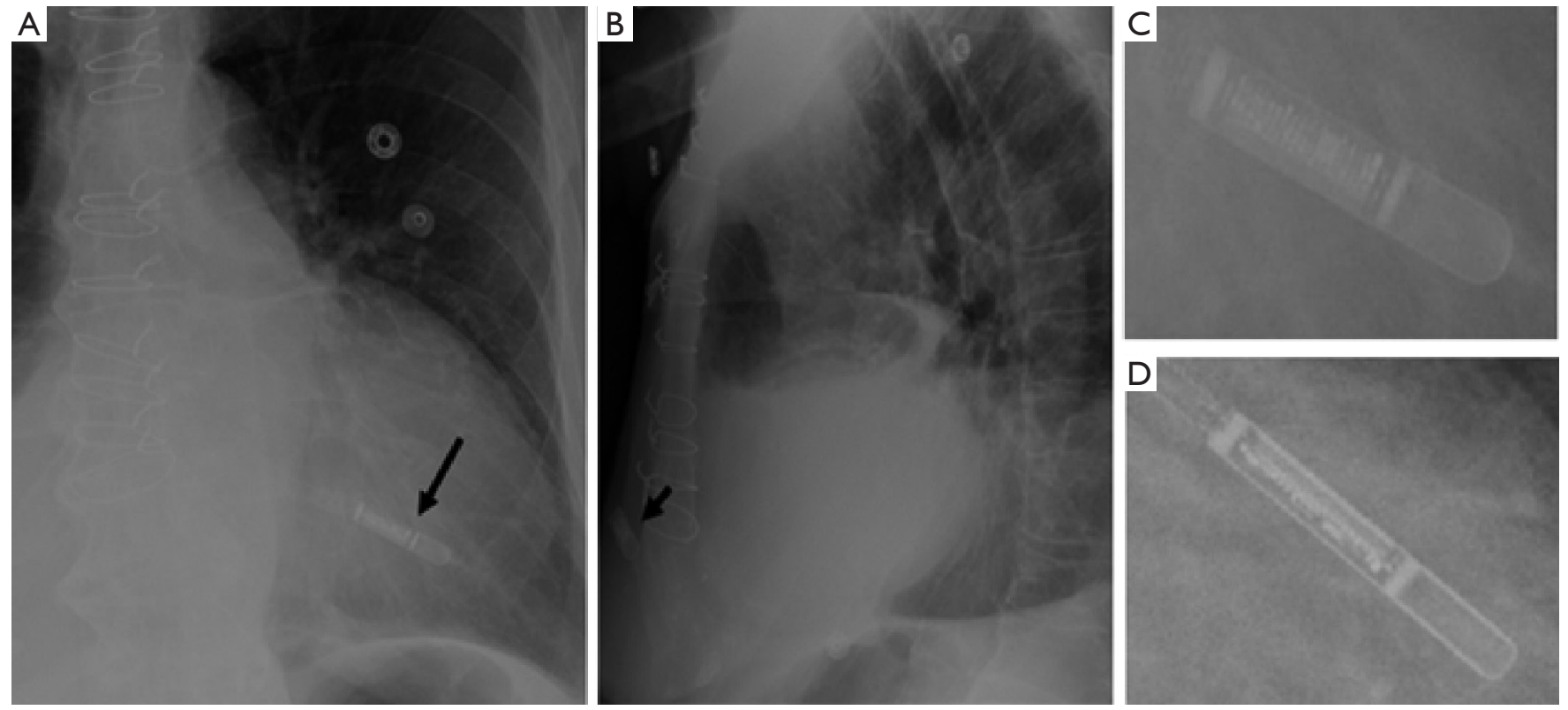

Figure 13 Loop recorder device. (A) Frontal and (B) lateral chest radiographs show the loop recorder device (arrow) which is similar to the leadless pacemaker device though easily differentiated on the lateral radiograph as the former projects within the subcutaneous chest wall. (C,D) Magnified images of the chest radiographs show different shapes and internal fabrics of the loop recorder devices.

\section{Ambulatory beart failure monitoring}

\section{Clinical indications}

Noninvasive measurements of PAP in patients with heart failure can be provided by implantable sensors, which are analogous to those of pulmonary artery catheterization and echocardiography.

\section{Device make and radiographic appearance}

The prototype device named CardioMEMS (St. Jude Medical or Abbott Inc., Chicago, IL) has an inductor coil and a pressure-sensitive capacitor placed in protective housing. It is deployed within a distal pulmonary artery (usually the left lower lobe), and secured in place with nitinol wire loops (Figure 14A,B). The device appears as a small radiopaque line with radiopaque dots on either end and may be missed on portable radiographs (Figure 14C,D). Magnified images and lateral CXRs are often better in depicting the characteristic figure-of-eight shape (Figure 14C, see insert).

\section{Mimic}

Without adequate awareness, this may be mistaken for embolized fragmented tip of a vascular catheter or guidewire.

\section{Device-specific complications}

Complications related to CardioMEMS include in-situ thrombosis and pulmonary artery injury resulting in hemoptysis (36).

\section{Other monitoring and pacing devices}

The Swan-Ganz catheter is used to monitor pulmonary capillary wedge pressure. It is usually inserted via a central vein (usually the right internal jugular) and traverses the right heart chambers with its tip lodged in a distal main pulmonary artery branch (usually the right).

Traditional cardiac pacemakers and defibrillators comprise an external pulse-generator or battery pack and transvenous leads which usually terminate in the right atrial appendage, right ventricle apex and/or coronary sinus. Newer Bundle of His leads positioned towards the 

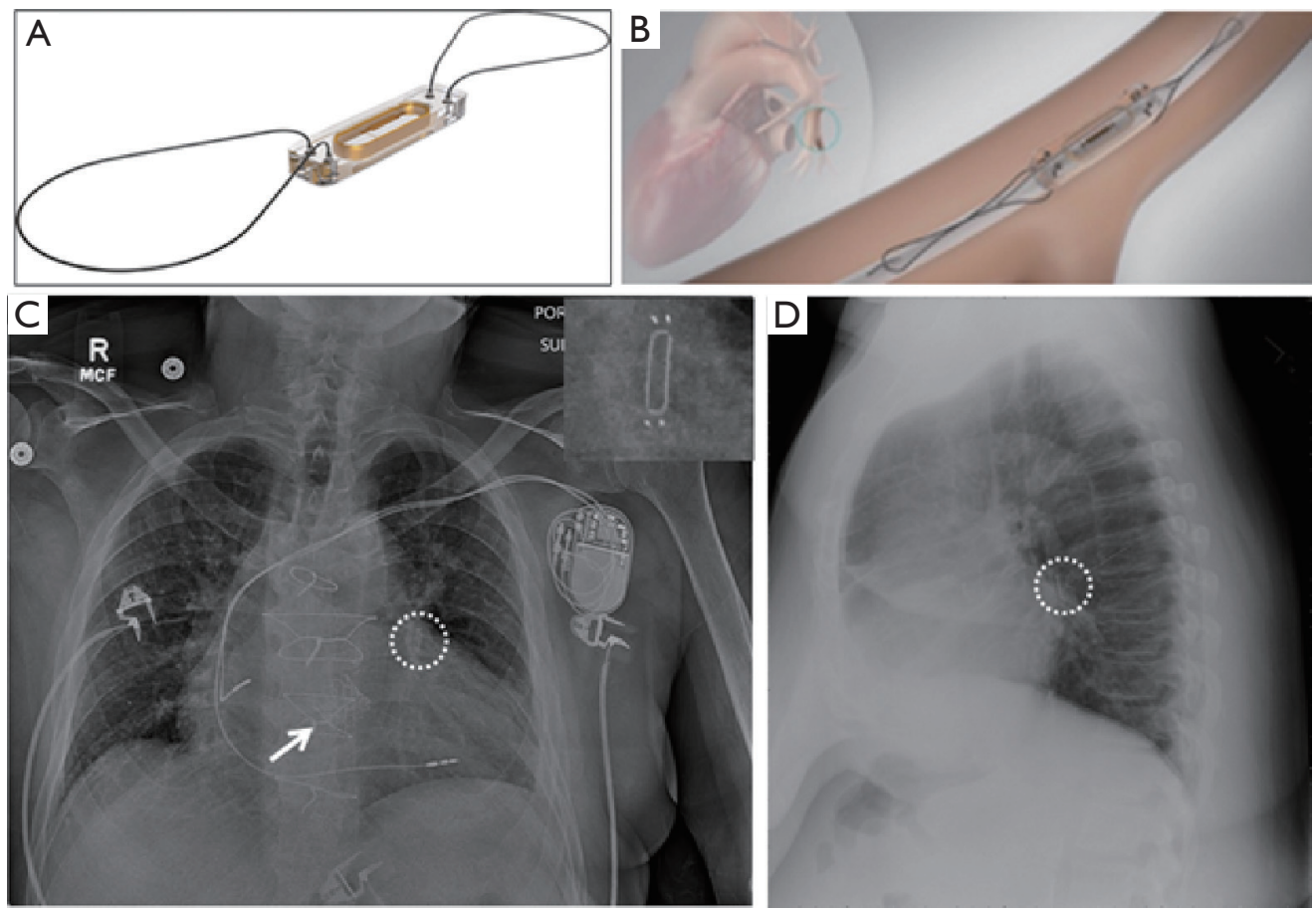

Figure 14 CardioMEMS Heart Failure System. (A,B) Photographs show the CardioMEMS device (Image courtesy of St Jude Medical) and the illustrated image of the device inside the inferior left pulmonary artery. (C) Frontal chest radiograph shows the CardioMEMS device (dashed circle) over the left lower lobe pulmonary artery with magnified image (insert). TAVR is noted (arrow). (D) Lateral chest radiograph shows the CardioMEMS device (dashed circle) over the lower lobe pulmonary artery.
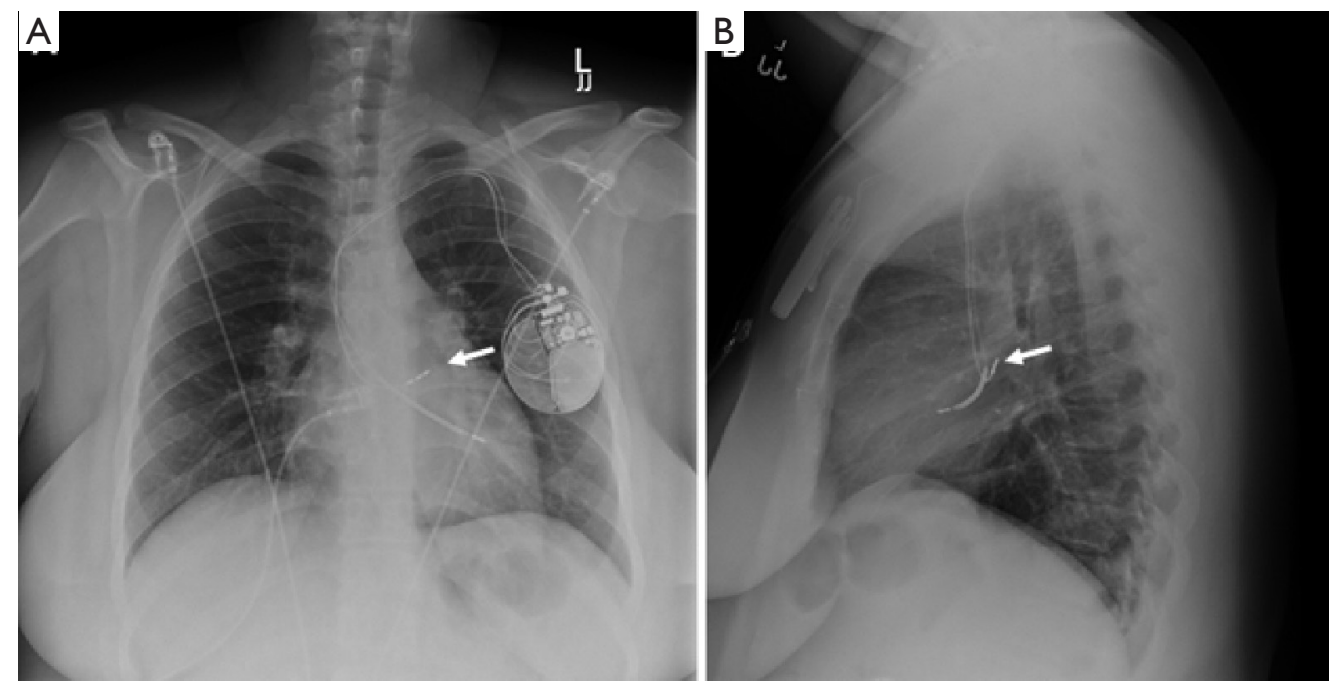

Figure 15 Bundle of His pacing lead. (A) Frontal and (B) lateral chest radiographs show a left chest wall cardiac defibrillator with 2 leads which terminate in the right ventricle and towards the Bundle of His. The Bundle of His lead is positioned towards the interventricular septum (arrow). 

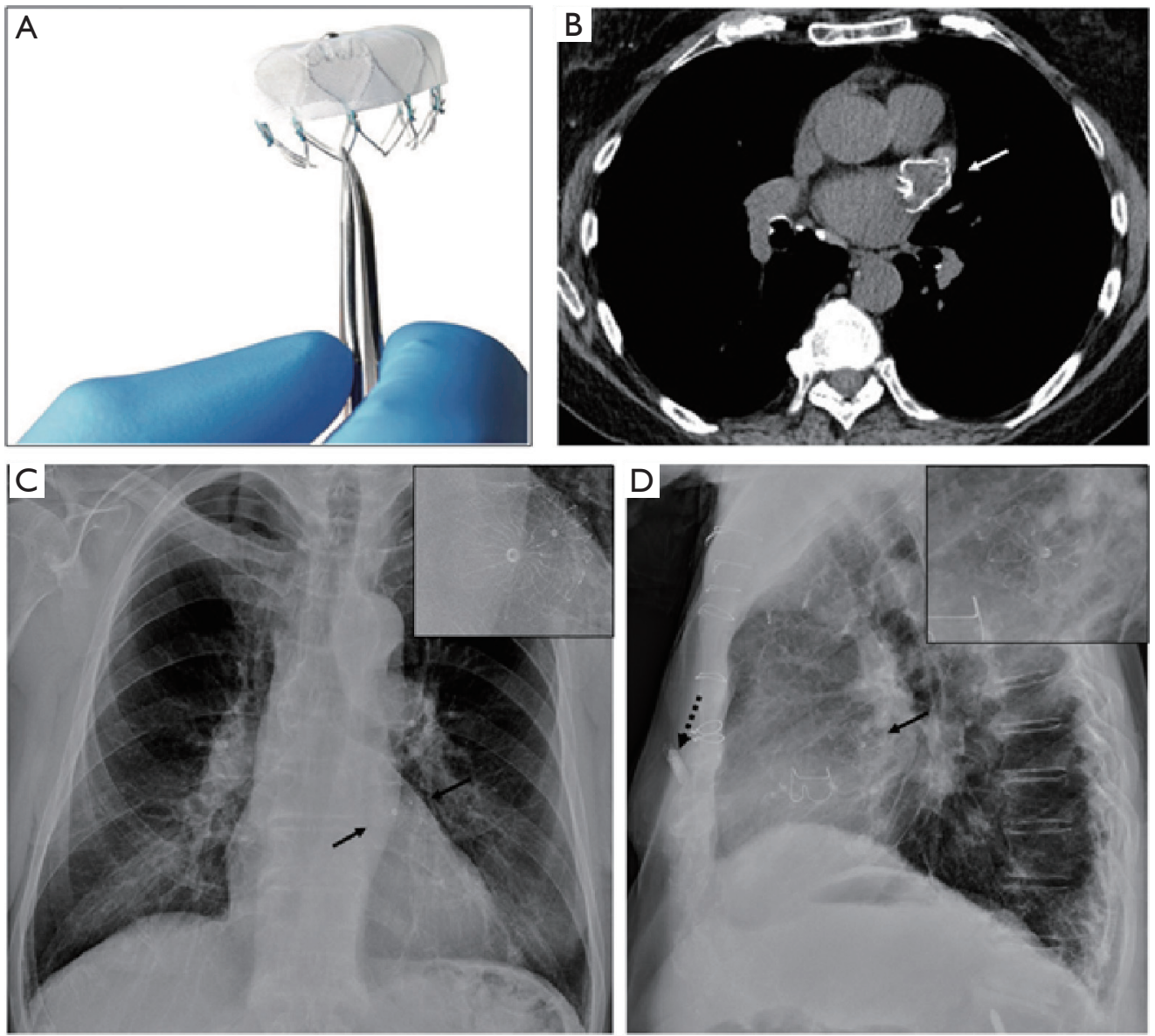

Figure 16 Watchman LAA closure device. (A) Photograph of a Watchman device shows the parachute shape and jellyfish-like appearance with nitinol frame (Image courtesy Boston Scientific). (B) Axial CT image shows the device in the expected position of LAA. (C) Frontal chest radiograph shows the LAA closure device (arrow) over the expected location of the LAA with magnified image (insert). (D) Lateral chest radiograph shows the LAA closure device (arrow) over the expected location of the LAA with magnified image (insert) and an anterior chest wall loop recorder (dashed arrows). LAA, left atrial appendage.

interventricular septum are uncommon and maybe mistaken for abnormal placement (Figure 15).

\section{Prevention of thromboembolism in the systemic and pulmonary circulation}

\section{Percutaneous left atrial appendage (LAA) closure devices}

\section{Clinical indications}

LAA closure devices are used in atrial fibrillation as alternatives to anticoagulation or in patients with contraindications to anticoagulation (37).

\section{Device make and radiographic appearance}

The Watchman device, Amplatzer Amulet, and Amplatzer Cardiac Plug are prototype LAA closure devices that are placed percutaneously. Watchman device (Atritech/Boston Scientific, Plymouth, MN) has a parachute shape and is often described as appearing jellyfish-like. It is composed of a self-expanding nitinol frame, with proximal face covered by a polyethylene terephthalate fabric (Figure 16A,B). The nitinol frame may be faintly visualized over the LAA location on frontal CXRs, and is often better depicted on lateral CXRs (Figure 16C,D). 


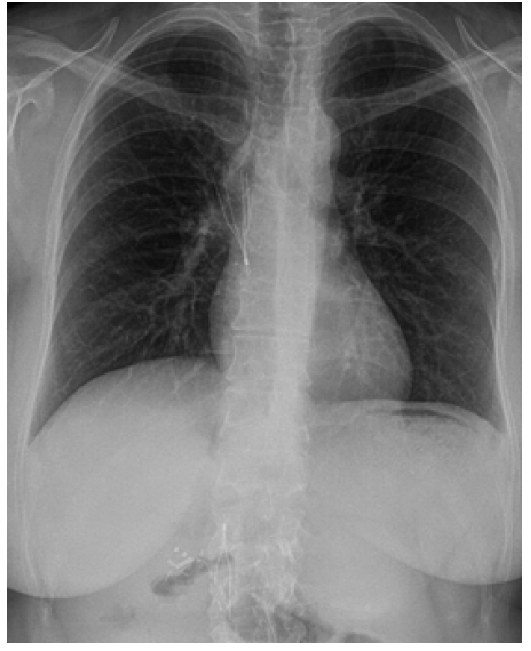

Figure 17 SVC and IVC filters. Frontal CXR shows an umbrellashaped device with spokes in the expected location of the SVC with the pointed tip of the device directed towards the SVCright atrial junction. A similar device is seen in the IVC with its tip directed towards the IVC-right atrial junction. CXR, chest radiography.

\section{Device-specific complications}

Lack of visualization in the expected LAA location on a radiograph may suggest device malposition or migration.

\section{Caval filters}

\section{Clinical indication}

SVC filters are less common than IVC filters, as incidence of lower extremity deep vein thrombosis (DVT) is way more common. An SVC filter is a safe and effective device for prevention of pulmonary embolism in patients with upper extremity DVT who have contraindication to anticoagulation. Swans and central venous catheters can be placed safely through the filter.

Device make and radiographic appearance of caval filters A vena cava filter is a small metal device shaped rather like the spokes of an umbrella, which projects over the SVC or IVC with its pointed tip directed towards the right atrium (Figure 17).

\section{Device specific complications of SVC filter}

Device migration, fracture, and dislodgement are uncommon potential complications.
Mimic

A deformed SVC stent may mimic SVC filter.

\section{Key features}

* TAVR/TAVI and TPVR devices share a common stentlike shape, and project over the expected locations of the aortic and pulmonic valves respectively.

* Mitral and tricuspid clips as the names imply, are short clip-like devices visualized over the mitral or tricuspid valve position (usually two placed closely parallel). Their shape may be mistaken for similar appearing endoscopic clips used for treatment of esophageal achalasia (per-oral endoscopic myotomy clips), though the latter are typically more midline on a frontal CXR (in keeping with the anatomic location of the thoracic esophagus).

* Clamshell-shaped septal occluder devices project over the inter-atrial and ventricular septum.

* A PDA occluder device also has a unique location in the aorto-pulmonary window and may come in one of several designs.

* A stent placed across an aortic coarctation is typically placed in the distal aortic arch/proximal descending aorta.

* The left ventricular partitioning device projects within the left ventricle cavity and has an umbrella or parachute-like design.

* Several catheter-based therapeutic and monitoring devices remain connected with long intravascular catheters or wires which extend outside the chest. These include the Impella, IABP, ECMO, PA catheters and various pacemaker/ICDs.

* Devices that overlie the right ventricular apex include the leadless cardiac pacemaker with the shape of a capsule, which can mimic a loop recorder on a frontalview CXR.

* Implanted loop-recorders are placed superficially within a subcutaneous pocket of the left anterior chest wall and can be clearly distinguished from the intra-cardiac leadless pacemaker (typically within the RV cavity) on a lateral CXR.

* A distinct, figure-of-eight shaped device which is used for monitoring PAPs in patients with heart failure (CardioMEMS), is usually placed within a distal branch of the left lower lobe pulmonary artery. Without heightened awareness of its presence, this small device may either be missed on the frontal radiograph 
(given its location and small size) or may simulate embolization of foreign body such as the fragmented tip of a vascular catheter.

* Several commercially available LAA closure devices are available, with the most common being the Watchman device, which has a parachute shape (jellyfish-like design).

* Caval filters in the IVC and SVC are easily identifiable given their characteristic location.

\section{MR compatibility of medical devices and implants}

Implanted transcatheter medical devices, particularly active implants or those with ferromagnetic material, can cause harm within the MRI environment. Standard MR safety terminologies include "MR safe," "MR unsafe," and "MR conditional". MR-safe devices are non-hazardous in all MRI environments (non-conducting, non-metallic, and non-magnetic items). MR-unsafe devices are considered to be contraindicated in any MR environments. An MRconditional device has manufacturer-specified operating conditions to allow MR imaging while minimizing risk such as the main magnetic field strength, maximum magnetic field gradient, and maximum specific absorption rate (SAR). Devices may be safe or conditional, but may impact MR image quality due to artefact (38).

Screening for these devices with CXR is an essential step prior to performing MRI. The MRI safety labeling of the discussed devices is summarized in Table-1. Best practice, whenever there is lack of documentation and certainty regarding a specific device's MR safety, is to review the device's information at www.mrisafety.com (30). Many manufacturers provide online MRI safety information and guidelines for their devices. In some cases, the device model numbers are needed to determine the degree of MRI compatibility and a note from the patient's surgeon may be required to confirm details, though not always feasible (39).

\section{Limitations}

An exhaustive list of all cardiothoracic devices is beyond the scope of this pictorial essay. As the title indicates, we have highlighted only the newer segment of the device market that are placed using a trans-catheter approach and excluded all surgically placed intra-cardiac devices as well as aortic stent-graft repairs. We have chosen to omit most of the traditional devices such as central lines, pacemakers and defibrillators, and very briefly discussed intra-aortic balloon

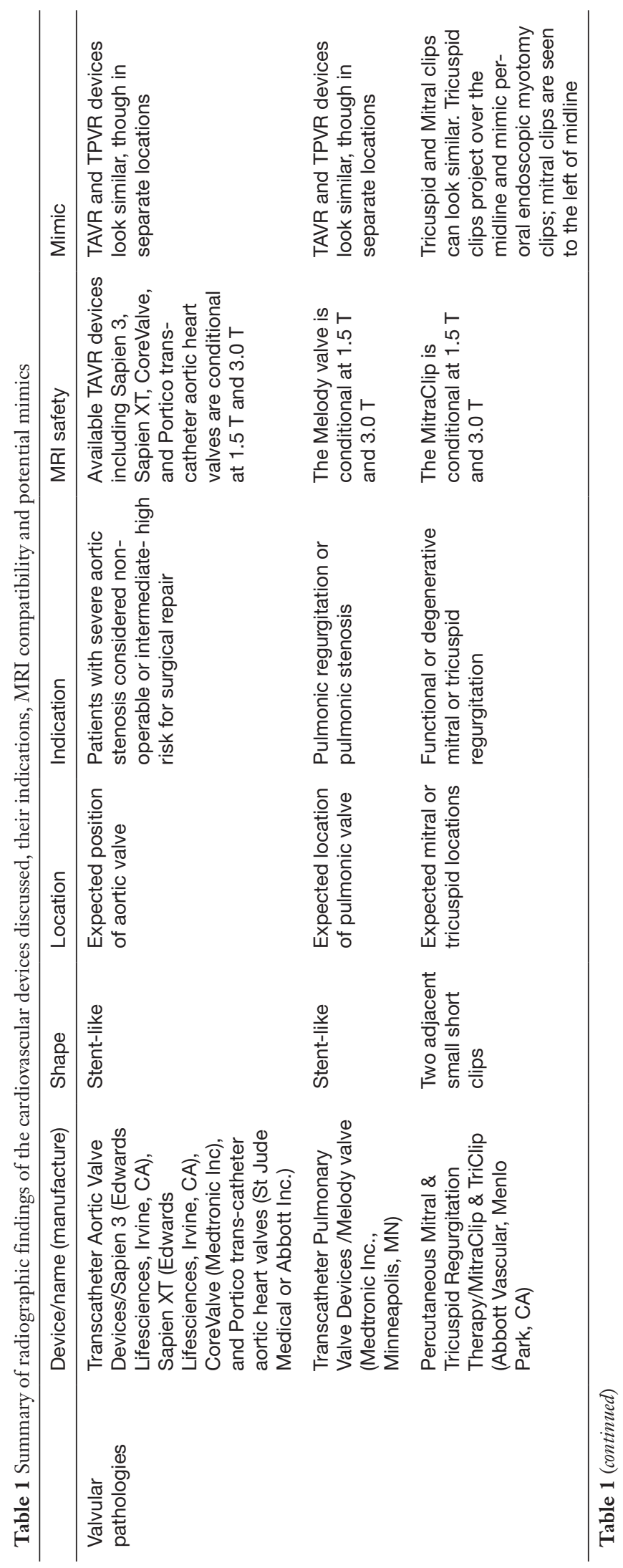




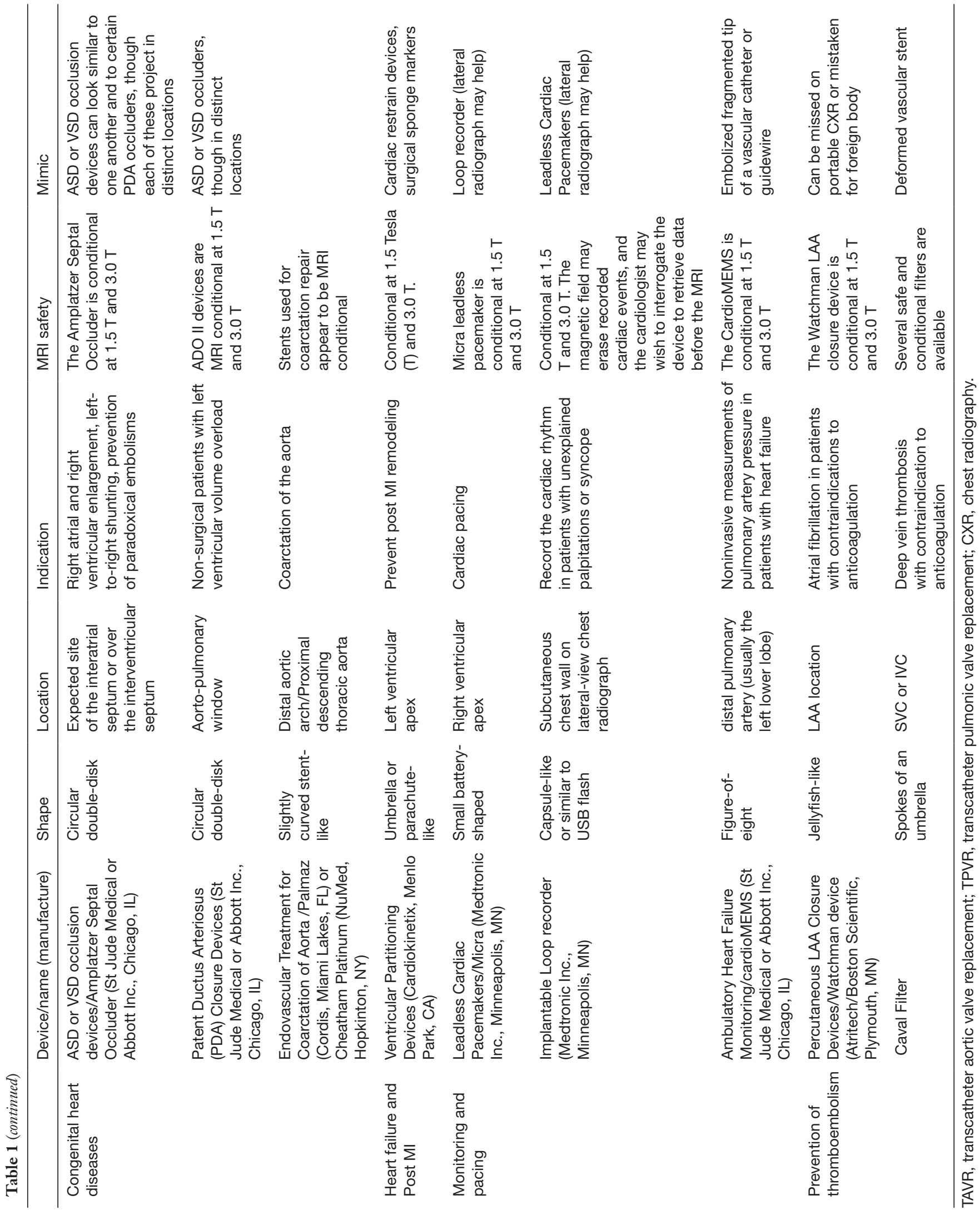


pumps and pulmonary artery catheters, as these have long since become mainstream and gained widespread familiarity in the imaging community. Lastly, we decided to leave out a discussion on coronary artery stents as these are much better evaluated using coronary angiography and almost always MRI compatible.

\section{Conclusions}

The radiographic findings of contemporary trans-catheter cardiovascular devices discussed, their indications, MRI compatibility, and potential radiographic mimcs are summarized in Table 1. Safety should always be confirmed and documented by reviewing device-specific information (including www.mrisafety.com if needed) prior to patient entry into the MR scanning area.

\section{Acknowledgments}

Funding: None.

\section{Footnote}

Conflicts of Interest: All authors have completed the ICMJE uniform disclosure form (available at http://dx.doi. org/10.21037/cdt-20-617). The authors have no conflicts of interest to declare.

Ethical Statement: The authors are accountable for all aspects of the work in ensuring that questions related to the accuracy or integrity of any part of the work are appropriately investigated and resolved.

Open Access Statement: This is an Open Access article distributed in accordance with the Creative Commons Attribution-NonCommercial-NoDerivs 4.0 International License (CC BY-NC-ND 4.0), which permits the noncommercial replication and distribution of the article with the strict proviso that no changes or edits are made and the original work is properly cited (including links to both the formal publication through the relevant DOI and the license). See: https://creativecommons.org/licenses/by-nc-nd/4.0/.

\section{References}

1. Fanari $Z$, Weintraub WS. Cost-effectiveness of transcatheter versus surgical management of structural heart disease. Cardiovasc Revasc Med 2016;17:44-7.
2. Kapadia SR, Leon MB, Makkar RR, et al. 5-year outcomes of transcatheter aortic valve replacement compared with standard treatment for patients with inoperable aortic stenosis (PARTNER 1): a randomised controlled trial. Lancet 2015;385:2485-91.

3. Leon MB, Smith CR, Mack MJ, et al. Transcatheter or Surgical Aortic-Valve Replacement in Intermediate-Risk Patients. N Engl J Med 2016;374:1609-20.

4. Sigakis CJG, Mathai SK, Suby-Long TD, et al. Radiographic Review of Current Therapeutic and Monitoring Devices in the Chest. Radiographics 2018;38:1027-45.

5. Pasic $M$, Unbehaun A, Buz S, et al. Annular rupture during transcatheter aortic valve replacement: classification, pathophysiology, diagnostics, treatment approaches, and prevention. JACC Cardiovasc Interv 2015;8:1-9.

6. Salgado RA, Budde RP, Leiner T, et al. Transcatheter aortic valve replacement: postoperative CT findings of Sapien and CoreValve transcatheter heart valves. Radiographics 2014;34:1517-36.

7. Hascoët S, Acar P, Boudjemline Y. Transcatheter pulmonary valvulation: current indications and available devices. Arch Cardiovasc Dis 2014;107:625-34.

8. Tretter JT, Friedberg MK, Wald RM, et al. Defining and refining indications for transcatheter pulmonary valve replacement in patients with repaired tetralogy of Fallot: Contributions from anatomical and functional imaging. Int J Cardiol 2016;221:916-25.

9. McElhinney DB, Hennesen JT. The Melody ${ }^{\circledR}$ valve and Ensemble ${ }^{\circledR}$ delivery system for transcatheter pulmonary valve replacement. Ann N Y Acad Sci 2013;1291:77-85.

10. Saremi F, Gera A, Ho SY, et al. CT and MR imaging of the pulmonary valve. Radiographics 2014;34:51-71.

11. Jilaihawi H, Hussaini A, Kar S. MitraClip: a novel percutaneous approach to mitral valve repair. J Zhejiang Univ Sci B 2011;12:633-7.

12. Feldman T, Kar S, Rinaldi M, et al. Percutaneous mitral repair with the MitraClip system: safety and midterm durability in the initial EVEREST (Endovascular Valve Edge-to-Edge REpair Study) cohort. J Am Coll Cardiol 2009;54:686-94.

13. Pighi M, Estevez-Loureiro R, Maisano F, et al. Immediate and 12-Month Outcomes of Ischemic Versus Nonischemic Functional Mitral Regurgitation in Patients Treated With MitraClip (from the 2011 to 2012 Pilot Sentinel Registry of Percutaneous Edge-To-Edge Mitral Valve Repair of the European Society of Cardiology). Am J Cardiol 2017;119:630-7. 
14. Nickenig G, Weber M, Lurz P, et al. Transcatheter edgeto-edge repair for reduction of tricuspid regurgitation: 6-month outcomes of the TRILUMINATE single-arm study. Lancet 2019;394:2002-11.

15. Moore J, Hegde S, El-Said H, et al. Transcatheter device closure of atrial septal defects: a safety review. JACC Cardiovasc Interv 2013;6:433-42.

16. Warnes CA, Williams RG, Bashore TM, et al. ACC/ AHA 2008 guidelines for the management of adults with congenital heart disease: a report of the American College of Cardiology/American Heart Association Task Force on Practice Guidelines (Writing Committee to Develop Guidelines on the Management of Adults With Congenital Heart Disease). Developed in Collaboration With the American Society of Echocardiography, Heart Rhythm Society, International Society for Adult Congenital Heart Disease, Society for Cardiovascular Angiography and Interventions, and Society of Thoracic Surgeons. J Am Coll Cardiol 2008; 52:e143-263.

17. Snijder RJ, Suttorp MJ, Berg JM, et al. Percutaneous closure of secundum type atrial septal defects: More than 5-year follow-up. World J Cardiol 2015;7:150-6.

18. Sudhakar P, Jose J, George OK. Contemporary outcomes of percutaneous closure of patent ductus arteriosus in adolescents and adults. Indian Heart J 2018;70:308-15.

19. Gruenstein DH, Ebeid M, Radtke W, et al. Transcatheter closure of patent ductus arteriosus using the AMPLATZER $^{\text {TM }}$ duct occluder II (ADO II). Catheter Cardiovasc Interv 2017;89:1118-28.

20. Boudjemline Y. The new Occlutech(ㅅ) patent ductus arteriosus occluder: Single centre experience. Arch Cardiovasc Dis 2016;109:384-9.

21. Azhar AS, Abd El-Azim AA, Habib HS. Transcatheter closure of patent ductus arteriosus: Evaluating the effect of the learning curve on the outcome. Ann Pediatr Cardiol 2009;2:36-40.

22. Forbes TJ, Gowda ST. Intravascular stent therapy for coarctation of the aorta. Methodist Debakey Cardiovasc J 2014;10:82-7.

23. Tyagi S, Singh S, Mukhopadhyay S, et al. Self- and balloon-expandable stent implantation for severe native coarctation of aorta in adults. Am Heart J 2003;146:920-8.

24. Ledesma M, Alva C, Gómez FD, et al. Results of stenting for aortic coarctation. Am J Cardiol 2001;88:460-2.

25. Pilla CB, Fontes VF, Pedra CA. Endovascular stenting for aortic coarctation. Expert Rev Cardiovasc Ther 2005;3:879-90.
26. Marshall AC, Perry SB, Keane JF, et al. Early results and medium-term follow-up of stent implantation for mild residual or recurrent aortic coarctation. Am Heart J 2000;139:1054-60.

27. Konstam MA, Kramer DG, Patel AR, et al. Left ventricular remodeling in heart failure: current concepts in clinical significance and assessment. JACC Cardiovasc Imaging 2011;4:98-108.

28. Costa MA, Pencina M, Nikolic S, et al. The PARACHUTE IV trial design and rationale: percutaneous ventricular restoration using the parachute device in patients with ischemic heart failure and dilated left ventricles. Am Heart J 2013;165:531-6.

29. Costa MA, Mazzaferri EL, Jr., Sievert H, et al. Percutaneous ventricular restoration using the parachute device in patients with ischemic heart failure: three-year outcomes of the PARACHUTE first-in-human study. Circ Heart Fail 2014;7:752-8.

30. Shellock FG. MRI Safety. Accessed June 24, 2020. Available online: http://www.mrisafety.com/

31. Mohamed I, Lau CT, Bolen MA, et al. Building a bridge to save a failing ventricle: radiologic evaluation of shortand long-term cardiac assist devices. Radiographics 2015;35:327-56.

32. Epstein AE, DiMarco JP, Ellenbogen KA, et al. 2012 ACCF/AHA/HRS focused update incorporated into the ACCF/AHA/HRS 2008 guidelines for device-based therapy of cardiac rhythm abnormalities: a report of the American College of Cardiology Foundation/American Heart Association Task Force on Practice Guidelines and the Heart Rhythm Society. J Am Coll Cardiol 2013;61:e6-75.

33. Knops RE, Tjong FV, Neuzil P, et al. Chronic performance of a leadless cardiac pacemaker: 1-year follow-up of the LEADLESS trial. J Am Coll Cardiol 2015;65:1497-504.

34. Reynolds DW, Ritter P. A Leadless Intracardiac Transcatheter Pacing System. N Engl J Med. 2016;374:2604-5.

35. Aguilera AL, Volokhina YV, Fisher KL. Radiography of cardiac conduction devices: a comprehensive review. Radiographics 2011;31:1669-82.

36. Rali AS, Shah Z, Sauer AJ, et al. Hemoptysis After CardioMEMS Implantation: Case Report and Review. Am J Case Rep 2018;19:382-5.

37. Holmes DR, Jr., Kar S, Price MJ, et al. Prospective randomized evaluation of the Watchman Left Atrial Appendage Closure device in patients with atrial 
fibrillation versus long-term warfarin therapy: the PREVAIL trial. J Am Coll Cardiol 2014;64:1-12.

38. Shellock FG, Woods TO, Crues JV 3rd. MR labeling information for implants and devices: explanation of

Cite this article as: Ghosh S, Abozeed M, Bin Saeedan M, Raman SV. Chest radiography of contemporary trans-catheter cardiovascular devices: a pictorial essay. Cardiovasc Diagn Ther 2020;10(6):1874-1894. doi: 10.21037/cdt-20-617 terminology. Radiology 2009;253:26-30.

39. Tsai LL, Grant AK, Mortele KJ, et al. A Practical Guide to MR Imaging Safety: What Radiologists Need to Know. Radiographics 2015;35:1722-37. 